\title{
DESCRIPCIÓN ANATÓMICA DE LA VASCULATURA ARTERIAL CAROTIDEA-CEREBRAL EN EL GALLO DOMESTICO GALLUS GALLUS LINNAEUS (AVES: GALLIFORMES: PHASIANIDAE)
}

\author{
Antonio Verduzco Mendoza, Emilio Arch Tirado, \\ María Elena Contreras FigueroA, Jaime LEYbón IbARRA, \\ Alfonso AlFARo RodrígUeZ y Juan LICONA BONILLA \\ Instituto Nacional de Rehabilitación. Avenida México-Xochimilco $\mathrm{N}^{\circ} 289$, \\ Colonia Arenal de Guadalupe, Del. Tlalpan, 14389 México, D. F., MÉXICO.
}

cnrverduzco@hotmail.com, earch@inr.gob.mx, elenacnr@yahoo.com.mx, jaleybon@hotmail.com, alfa1360@yahoo.com.mx, cherry_net13@hotmail.com

Verduzco M., A., E. Arch T., M. E. Contreras F., J. Leybón I., A. Alfaro R., y J. Licona B. 2009. Descripción anatómica de la vasculatura arterial carotidea-cerebral en el gallo Gallus gallus Linnaeus (Aves: Galliformes: Phasianidae). Acta Zoológica Mexicana (n. s.), 25(3): 465-477.

RESUMEN. Se describe la vasculatura arterial carotídea-cerebral del gallo doméstico (Gallus gallus Linnaeus). Se utilizaron 10 gallos de $2.5 \mathrm{~kg}$ de peso, estirpe Rhode Island, los cuales fueron sometidos a la técnica de conservación replesión vascular. Se concluyó que la anastomosis intercarotídea, la formación de la arteria basilar y la ausencia de las arterias vertebrales son los componentes más sobresalientes de la vasculatura cefálica. Se propone estandarizar la nomenclatura de las diversas estructuras anatómicas y vasculares ligadas a la vasculatura arterial carotídea-cerebral ya que son confusas en las diversas publicaciones.

Palabras clave: Gallo doméstico, arterias, encéfalo, anatomía, disección

Verduzco M., A., E. Arch T., M. E. Contreras F., J. Leybón I., A. Alfaro R., \& J. Licona B. 2009. Anatomical description of the vessels arterial carotid-cerebral in domestic chicken Gallus gallus Linnaeus (Aves: Galliformes: Phasianidae). Acta Zoológica Mexicana (n. s.), 25(3): 465-477.

ABSTRACT. We describe the carotid-cerebral arterial vasculature of the domestic rooster (Gallus gallus Linnaeus). Ten cocks race Rhode Island of $2.5 \mathrm{~kg}$ of weight were used, which were subjected to vascular replesion conservation technique. It was concluded that anastomosis intercarotidea, the formation of the basilar artery and the absence of the vertebral arteries are the most significant components of the cephalic vasculature. It is proposed to standardize the nomenclature of the arteries of the brain-stem carotid arterial because of the confusing terminology used by various authors.

Key words: Domestic Chicken, arteries, brain, anatomy, dissection

Recibido: 14/05/2008; aceptado: 25/06/2009. 


\section{INTRODUCCIÓN}

Aún cuando las aves se han clasificado en una gran cantidad de órdenes taxonómicos de acuerdo con sus características filogenéticas, las diferencias anatómicas en las aves son relativamente pequeñas (Morales-Martínez et al. 2002). Glenny (1945a, 1945b, 1951a, 1951b, 1953) realiza una amplia descripción de la vasculatura arterial en la región del corazón en diferentes órdenes de aves y señala diferencias anatómicas entre las aves de cada orden. Abdalla \& King (1977), describieron las arterias esofagotraqueobronquiales en diversas especies de aves como gansos, patos y pavos, observando diferencias anatómicas entre especies relacionadas principalmente con aspectos de simetría y de irrigación sanguínea hacia órganos como: traquea, siringe, esófago y bronquios. Por su parte Abdel-Magied \& King (1978) realizan una precisa disección del área cardiaca y torácica, y esquematizan claramente estructuras vasculares, nerviosas, vasos linfáticos, ganglios, etc. en aves de corral (Gallus gallus). Holliday et al. (2006) describe la vasculatura cerebral en flamencos (Phoenicopterus ruber) mediante el uso de la tomografía computarizada de alta resolución, esta técnica permitió la identificación exacta de las estructuras vasculares cerebrales. Menciona que los flamencos comparten un número similar de patrones vasculares con otras aves, incluyendo la asimetría de la arteria cerebral caudal y las complejas variedades de sistemas vasculares como la red oftálmica, sin embargo señala que existen diferencias particulares en los patrones de ramificación de algunos vasos como la arteria occipital. Finalmente concluyen que los patrones vasculares en los Flamingos son semejantes a los de otras especies de aves y que las estructuras vasculares cefálicas en las aves han evolucionado de forma conservadora. Otros estudios de anatomía vascular en aves fueron llevados a cabo por Pettit et al.(1981) quien comparó la Red Mirable Oftálmica (RMO) en 6 diferentes especies de aves acuáticas de Hawai: Procellariiformes, Charadriformes y Pelecaniformes; en todas las aves se encontró que la RMO esta formada por la continuación de la Arteria Oftálmica Externa (AOE) que deriva de la Arteria Carótida Común (ACC), además se determinó que los patrones morfológicos de la RMO son semejantes entre estos grupos de aves marinas. También describe la existencia de tres tipos morfológicos de Anastomosis Intercarotídea, ya sea en forma de " $H$ ", de "X" y la mixta "X-H". La descripción de esta estructura es importante ya que interviene de manera importante en la distribución del flujo sanguíneo a nivel cerebral. Recientes hallazgos fueron descritos por (Aslan et al. 2006) quien detalla la vasculatura arterial en aves domésticas y de ornato, entre estas se encuentra una ave originaria de Turquía, el Denizli rooster (G. gallus domestica), gallo doméstico, faisán, faisán plateado, ganso y pavo. La anastomosis en forma de "H" se encontró en el Denizli rooster, gallo domestico, faisán y faisán plateado, mientras que en la anastomosis cruzada como una forma "X", fue observada en el pavo y el ganso. Existe también una anastomosis extracraneal en la parte craneal de la arteria oftalmotemporal y la arteria carótida 
cerebral en el Denizli rooster, gallo doméstico, faisán y faisán plateado, por otra parte se describe que la Arteria Basilar (AB) es formada por la continuación de la rama caudal derecha en el Denizli rooster, gallo doméstico y ganso. Mientras que la arteria basilar en el faisán, faisán plateado y pavo, fue formada por la rama caudal izquierda. Aunque en la literatura se menciona que la vasculatura aviar es semejante a la de los mamíferos (Jessen C 2001, Baumel \& Gerchman 1968), existen diferencias estructurales de importancia que deben describirse y considerarse, sobre todo para aquellos investigadores que pudieran considerar a las aves como modelos experimentales en estudios de hipoxia-isquemia, aprendizaje, así como en analogías vasculares entre aves y mamíferos, anastomosis intercarotídea y polígono de Willis respectivamente. Existen en la literatura diversas fuentes de consulta, libros (Ghetie et al. 1981, McLelland 1992, Sisson \& Grossman 1983) y publicaciones científicas (Pettit et al.1981, Aslan et al. 2006), las cuales no son lo suficientemente representativas de la vasculatura cerebral en el gallo doméstico.

El objetivo del presente trabajo, es describir la vasculatura arterial carotídeacerebral del gallo doméstico, con la finalidad de detallar su irrigación y describirla desde un punto de vista filogenético para su posible utilización en estudios comparativos

\section{MATERIAL Y MÉTODOS}

Para el estudio de la vasculatura arterial carotídea y cerebral se utilizaron 10 gallos de $2.5 \mathrm{~kg}$, estirpe Rhode Island. Se procedió a anestesiar a los gallos con clorhidrato de ketamina a dosis de $50 \mathrm{mg} / \mathrm{kg}$ vía intramuscular, se canalizó por vía endovenosa en la vena braquial derecha se aplicó heparina a dosis de 250 unidades $/ \mathrm{kg}$. con el propósito de eliminar la mayor cantidad de sangre del torrente sanguíneo para que al introducir el caucho de silicón se facilitara el proceso de perfusión. Posteriormente los animales se sacrificaron de forma humanitaria mediante sobredosis anestésica de Clorhidrato de ketamina.

Técnica de repleción vascular. La preparación del caucho de silicón consiste en la mezcla del caucho con un aditivo catalizador y un colorante orgánico color rojo marca poliformas ® codigo 56306206, al realizar la mezcla se inició el proceso de catalización lo que permite su aplicación aproximadamente por 20 minutos antes de la polimerización del producto. La perfusión se realizó vía arterial introduciendo un catéter en la arteria carótida común izquierda que corre paralelo a la musculatura cervical, de esta forma con una pistola para silicón se ejerce presión para prefundir el caucho de silicón por vía arterial pigmentándose la vasculatura arterial en su totalidad. Los gallos se dejaron reposar por $24 \mathrm{hrs}$ y posteriormente se iniciaron las disecciones de las estructuras anatómicas vasculares, desde los grandes vasos arteriales como la arteria carótida común hasta la microvasculatura en corteza cerebral. 
Para la localización de la vasculatura arterial, se retiró la piel y se disectó la musculatura en la parte medial ventral del cuello del ave, se identificó el canal muscular por donde emergen las arterias carótidas comunes izquierda (ACCI) y derecha (ACCD), y se dió seguimiento a la ACCI, debido a que fue la arteria canalizada para la perfusión.

Las disecciones se realizaron en los quirófanos de Cirugía Experimental del Instituto Nacional de Rehabilitación, utilizando instrumental especializado para microcirugía: Pinzas de disección RS-5130, pinzas hemostáticas Baby Halsted, tijeras Castroviejo RS-5650 y portagujas Castroviejo RS-6410. Las imágenes fotográficas se obtuvieron con la ayuda de una cámara Canon ® Power Shot SD750 DIGITAL ELPH DE 7.7 Mega Pixels y un microscopio estereoscópico quirúrgico Marca CARL-ZEISS 6615.

\section{RESULTADOS}

Descripción anatómica arterial. En el gallo doméstico, las arterias carótidas comunes ascienden por línea media ventral, inmersas en un surco formado por músculos de las vértebras cervicales, una vez que emergen del surco se dividen hacia el lado izquierdo y derecho en donde se derivan las distintas ramificaciones hacia las diferentes estructuras que van hacia cabeza y cuello (Fig. 1).

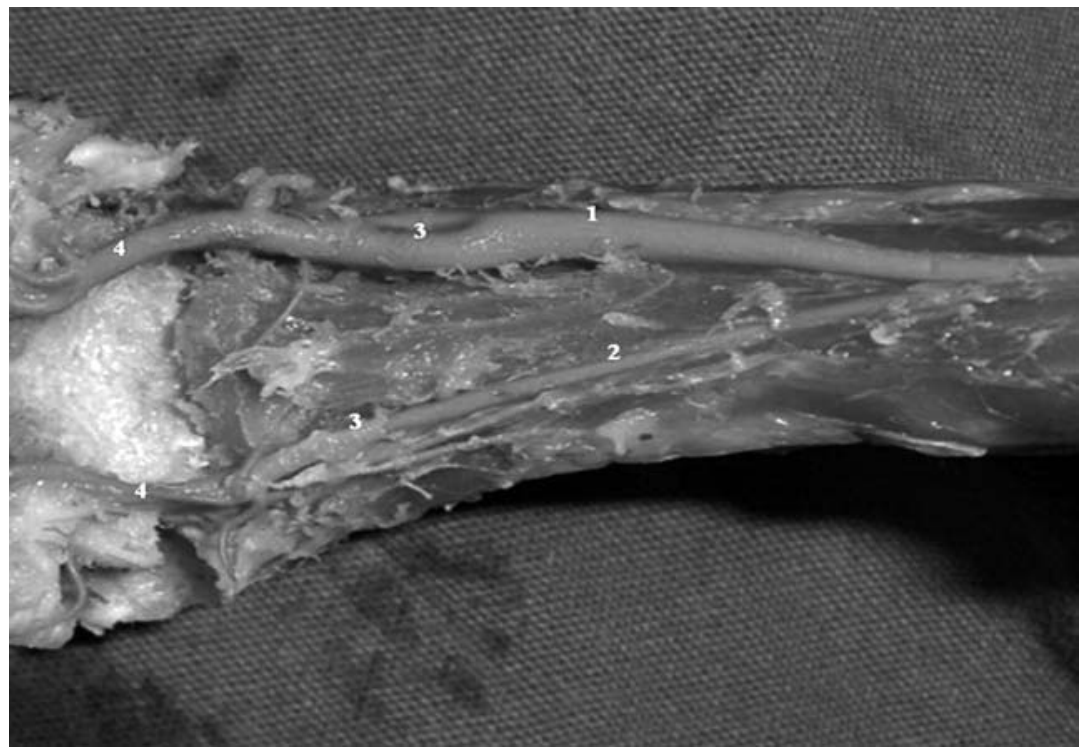

Figura 1. En la fotografía se observa el trayecto de la Arteria Carótida Común Izquierda-ACCI (1) y Arteria Carótida Común Derecha-ACCD (2). La bifurcación de las Carótidas Comunes (3) forma la

Arteria Carótida Interna-ACI, la cual se desplaza hacia la parte anterior y superior y la Arteria Carótida Externa (4) continúa su trayecto hacia craneal (4). 
La primer ramificación que surge de la arteria carótida común (ACC) es la rama correspondiente a la arteria carótida interna (ACI), la cual se desplaza de forma lateral y ascendente hacia el hueso temporal, en este punto sufre una flexión considerable en la fosa tímpano-auricular y se ramifica para formar la arteria oftálmica externa (AOE) que va a dar origen a la red oftálmica (RO) que se ubica de forma anterior al ojo (Fig. 2). La AOE atraviesa el oído medio por un canal dorsomedial a la ventana oval y emerge para subdividirse dentro de 4 ramas: temporal, supraorbital, oftálmica y infraorbital. Todas las ramas y la arteria alveolar interior contribuyen con vasos arteriales como componentes de la RO.

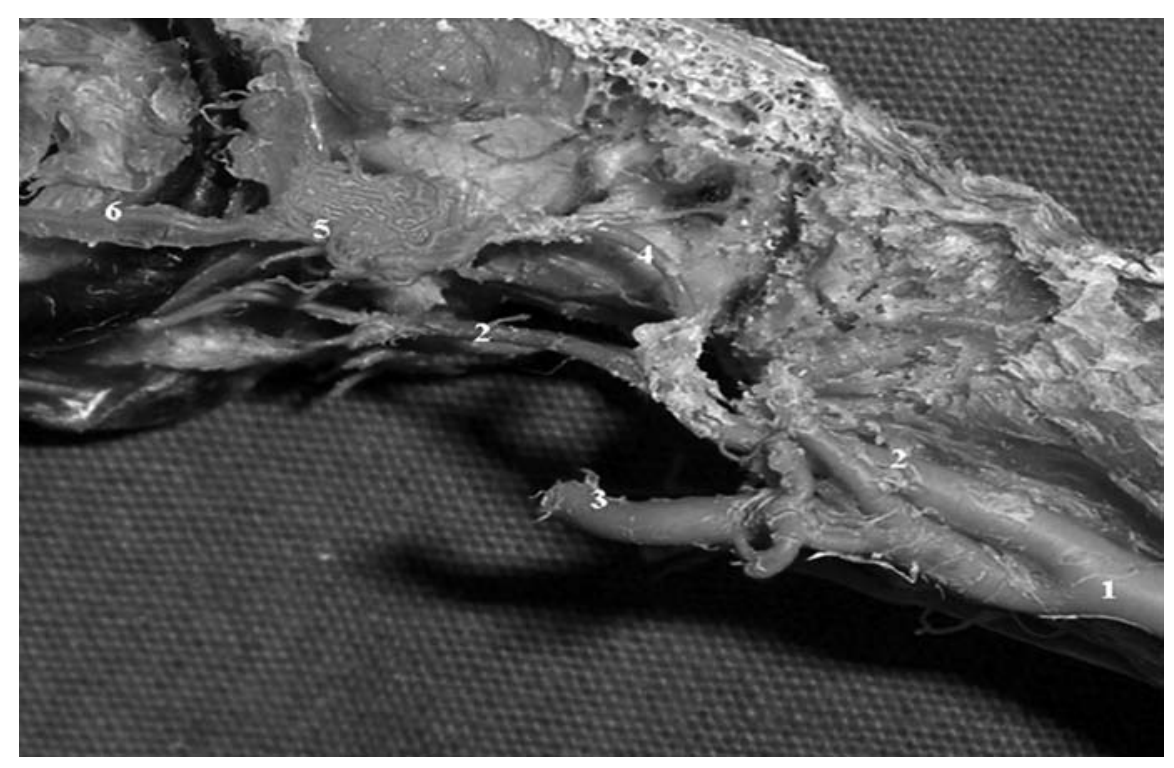

Figura 2. Nótese la bifurcación de la Arteria Carótida Común-ACC (1), la cual va a formar la Arteria Carótida Interna-ACI (2), y la Arteria Carótida Externa-ACE (3). La Arteria Oftálmica Externa-AOE (4) forma la Red Oftálmica-RO (5), también se identifica el trayecto de la Arteria Palpebral InferiorAPI (6).

En la figura 3, se observa que la ACI se divide en el borde de la fosa tímpanoauricular, para formar la AOE. La ACI se desplaza a través de un canal en la base del hueso basiesfenoides para formar en la parte medial la anastomosis intercarotídea (AI). Otra anastomosis, en esta área, está constituida extracranealmente por las arterias oftalmotemporal y carótida interna (ACI). 


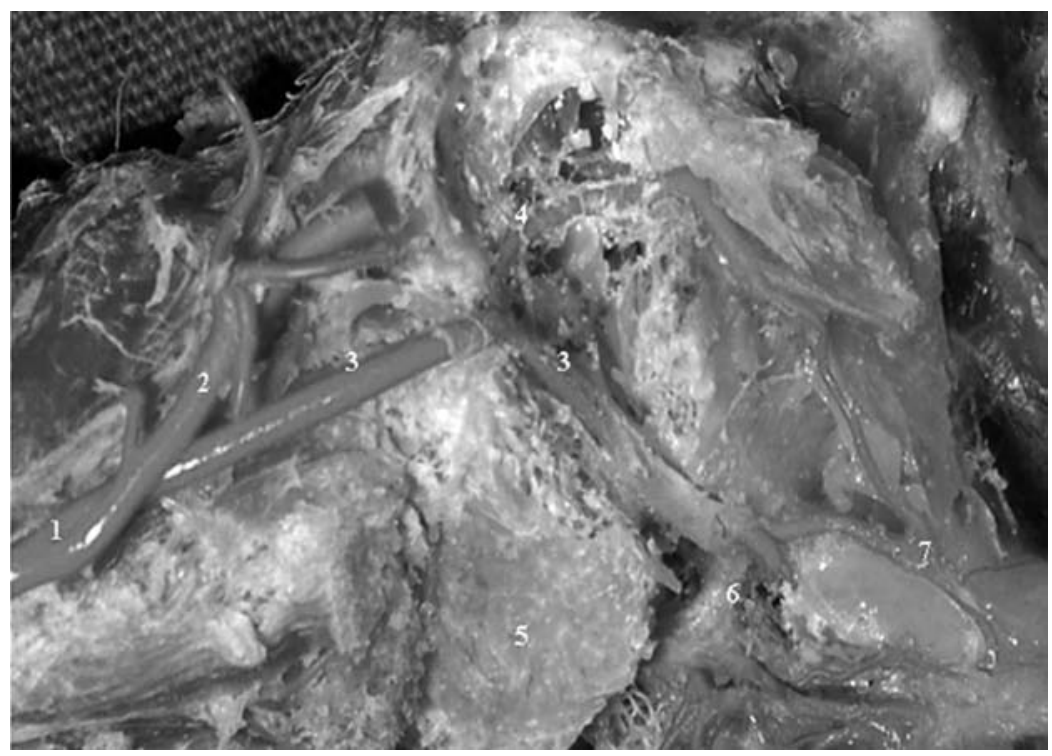

Figura 3. En la imagen se observa la Arteria Carótida Común-ACC (1), la Arteria Carótida ExternaACE (2) y la Arteria Carótida Interna-ACI (3), la cual se desplaza a través del hueso basiesfenoides

(5). Además se identifica la Anastomosis Intercarotídea-AI (6) y la anastomosis entre la Arteria

Oftalmotemporal y la ACI (7). Se muestra también la Arteria Oftálmica Externa-AOE (4).

Arteria carótida común y sus principales ramas. La ACC se aproxima al cráneo y se divide en ACI y ACE, sobre la ACE se forma un tronco arterial del que derivan diversos vasos que irrigan a órganos y músculos de la región cervical y cefálica, de esta manera surgen las arterias Occipital (AO), Comes Nervi Vagi (ACNV), Laringotraqueoesofágica (ALTE) y Músculo Cutánea (AMC). Mientras tanto, la ACE después de bifurcarse con la $\mathrm{ACI}$, continúa su trayecto para formar otros vasos arteriales como las arterias auricular (AA), facial (AF), lingual (AL) y pterigoidea (AP). En la parte más craneal de la $\mathrm{ACE}$, en relación con los músculos mandibulopalatinos y músculos entotimpánicos, se origina la Arteria Mandibular (AM) y las Arterias Palatinas (APal), las cuales se dividen en rama medial y rama lateral (Fig. 4).

Una de las estructuras características de la vasculatura cerebral en las aves es la Anastomosis Intercarotídea (AI), la cual se forma por la unión de las arterias carótidas internas izquierda y derecha, la AI permite que la sangre arterial se mezcle en este punto anatómico, esta peculiaridad de la fisiología aviar es factible debido al considerable calibre del vaso que forma la anastomosis (Fig.5). Se considera que las Arterias Carótidas Cerebrales son la continuación de las Arterias Carótidas Internas, las cuales atraviesan la cavidad craneal medialmente en su patrón intraoseo (segmento intraesfenoideo) Las Arterias Carótidas Internas izquierda y derecha en la 


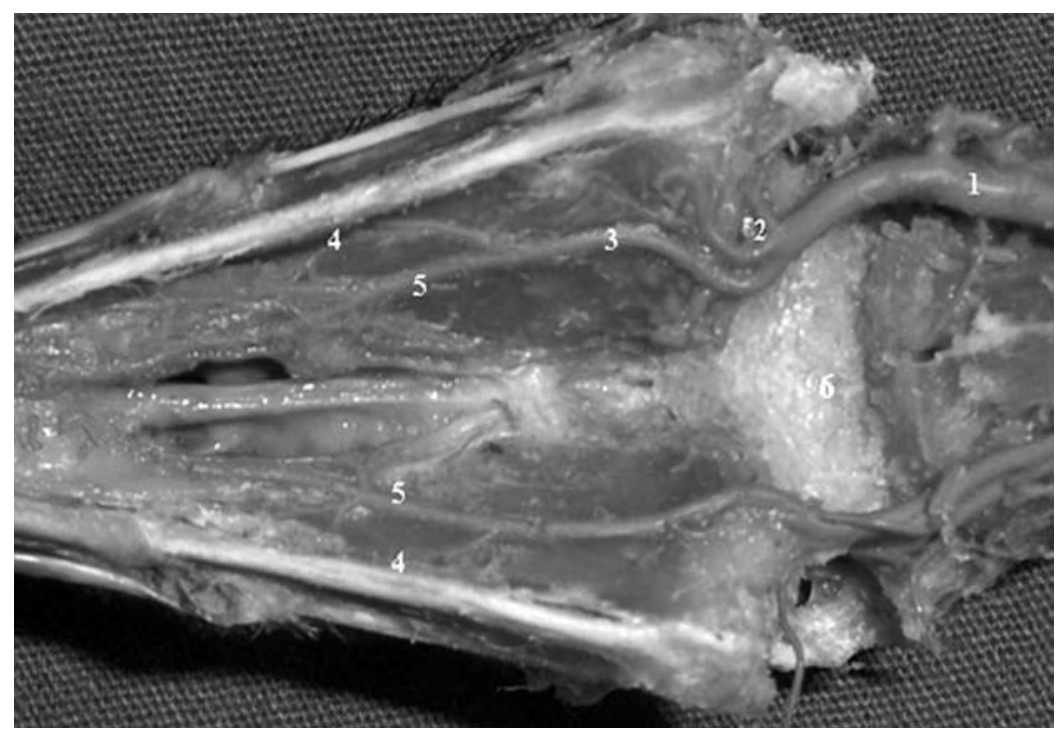

Figura 4. Arterias del paladar. La arteria carótida externa-ACE (1) continúa un trayecto craneal para derivar la arteria pterigiodea-AP (2) y las arterias: palatina-APal (3) laterales (4) y mediales (5). Obsérvese la estructura porosa del hueso basiesfenoides (6).

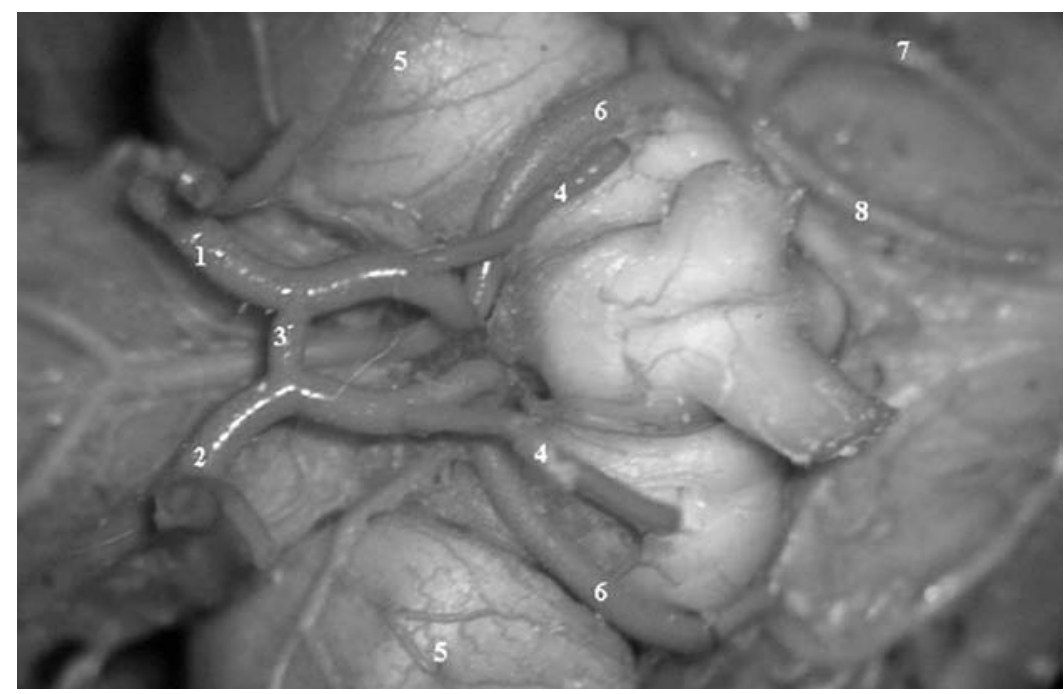

Figura 5. Obsérvese la vasculatura en la base del cerebro, se identifica la Arteria Carótida Interna Derecha-ACID (1), la Arteria Carótida Interna Izquierda-ACII (2), y la Anastomosis Intercarotidea-AI (3). También está representada la Arteria Oftálmica-AO (4), el Lóbulo Optico-LO (5), la Rama Craneal de la Arteria Carótida Interna-RCACI (6), la Arteria Cerebral Media- ACM (7) y la Arteria Cerebral Oral-ACO (8). 
parte inmediata caudal al quiasma óptico se aproximan una a otra para comunicarse por medio de la AI. Las Arterias Cerebrales se dividen para formar la rama craneal que irriga a los lóbulos craneales y mediales del cerebro y la rama caudal que perfunde hacia cerebelo y tallo cerebral.

En el caso del gallo doméstico, la rama caudal derecha forma la Arteria Basilar (AB) la cual desciende por la parte ventral de tallo cerebral proyectando pequeñas ramificaciones hacia diferentes estructuras que incluyen el puente (Arterias pontinas), la medula oblongada y el cerebelo (Fig. 6). La Arteria Cerebelar Ventral Aboralis (ACVA), la cual deriva de la Arteria Basilar (AB) para irrigar gran parte del parenquima cerebelar, se distingue por la presencia de una flexura lateral inmersa en el hueso temporal (Fig. 7). La Arteria Cerebelar Ventral Oral (ACVO), irriga también la parte anterior y ventral del cerebelo. En el área pontocerebelosa del gallo doméstico, puede identificarse la $\mathrm{AB}$, la cual derivó de la rama caudal derecha. La AB forma a su vez la ACVA y a la Arteria Espinal Ventral (AEV). Las Arterias Vertebrales no forman parte de la irrigación cerebral, como sucede en los mamíferos (Fig. 8).

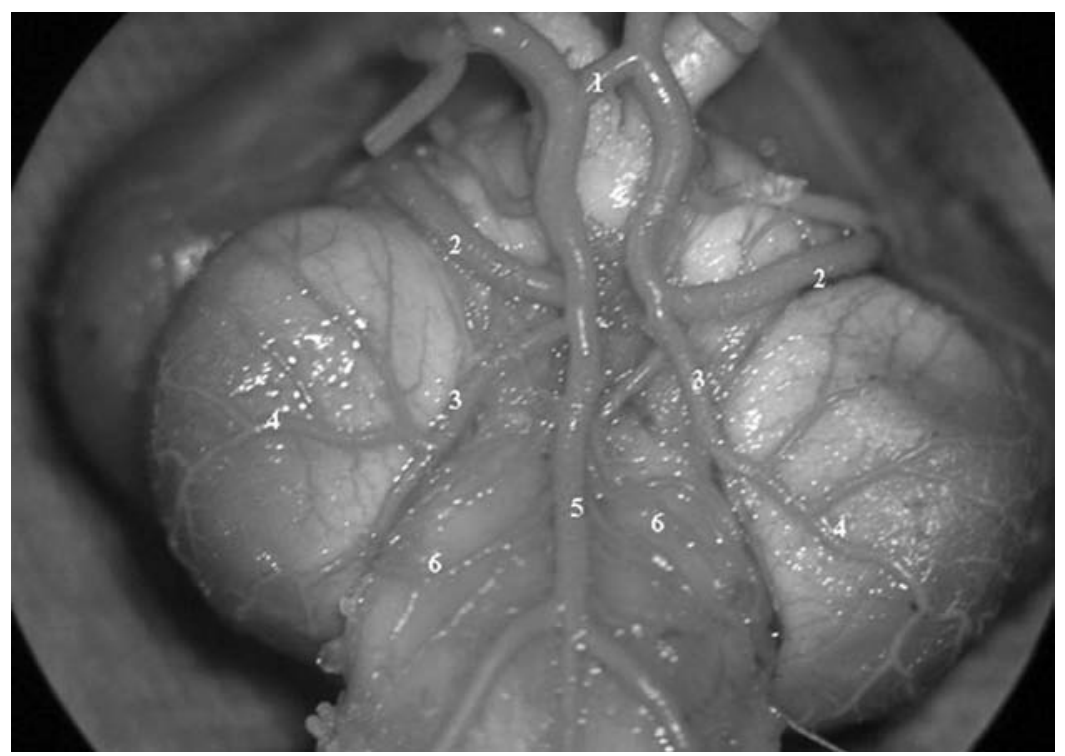

Figura 6. Vista ventral y posterior, nótese la Anastomosis Intercarotídea-AI(1), la Rama Craneal de la Arteria Carótida Interna-RCrACI (2), Rama Caudal de la Arteria Carótida Interna-RCaACI (3), la Arteria Tecti Optici Ventralis-ATOV(4), la Arteria Basilar-AB (5)y su origen de la Rama Caudal Derecha- (RCD) así como las Arterias Pontinas-AP (6). 


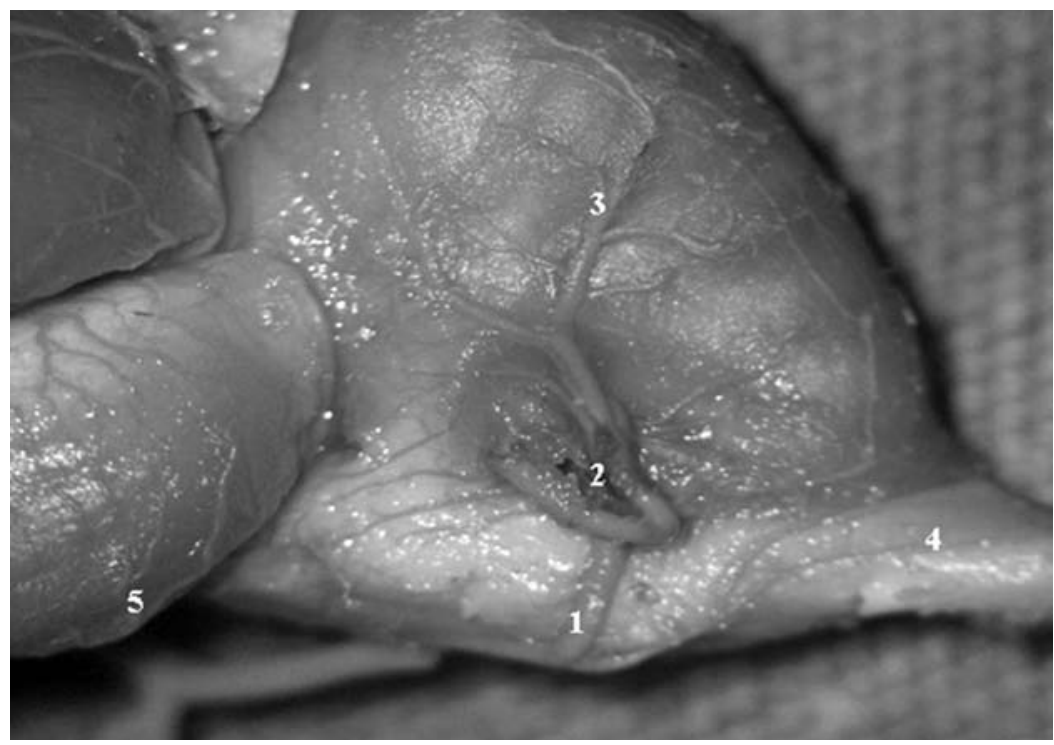

Figura 7. En la Imagen se identifica a la Arteria Cerebelar Ventral Aboralis-ACVA (1), la flexura lateral de la ACVA, la ramificación sobre el parénquima cerebelar de la ACVA (3), la Arteria Espinal Dorsal-AED(4) y Arteria Tecti Optici Ventralis-ATOV (5).

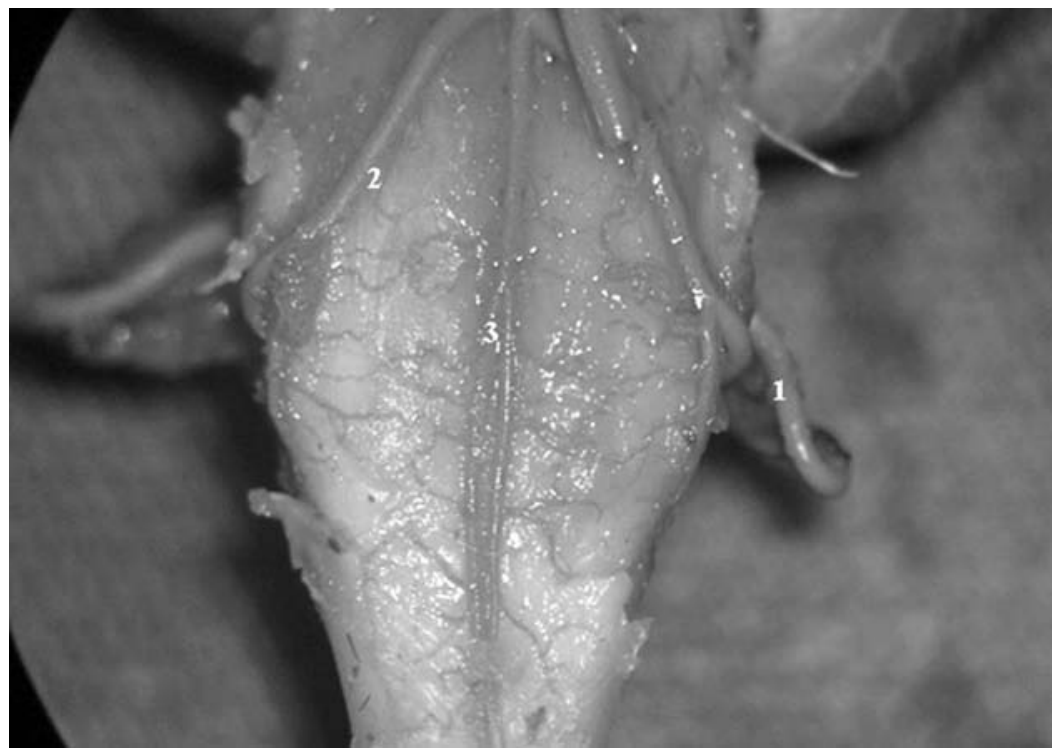

Figura 8. Se observa la flexura lateral (1), la Arteria Cerebelar Ventral Aboralis-ACVA (2) y las Arterias Espinales Ventrales-AEV que no deben confundirse con las ausentes arterias vertebrales (3). 
Verduzco et al.: Anatomía arterial carotidea-cerebral en Gallus gallus

\section{DISCUSIÓN}

En el presente trabajo se describió la vasculatura arterial carotídea-cerebral del gallo doméstico. La anastomosis intercarotídea (AI), la formación de la arteria basilar dada por la arteria cerebral posterior y la ausencia de las arterias vertebrales, son los componentes anatómicos sobresalientes de la vasculatura arterial cerebral en esta especie.

Al parecer, la AI es un rasgo anatómico exclusivo de las aves (Baumel \& Gerchman 1968), relacionado indudablemente con mecanismos evolutivos, que repercuten en eventos fisiológicos relacionados con la hemodinamia cerebral, homeostasis, mecanismos de adaptación y fisiología del vuelo. Algunos autores como (Pettit et al.1981) han descrito diferencias morfológicas de la AI, describiendo las formas en "H", "X" y "X-H". Con respecto a las analogías que comparten aves y mamíferos, cabe mencionar que las aves no poseen un sistema arterial cerebral tan complejo como lo es el Polígono de Willis, presente en los mamíferos, sin embargo la AI, como se puede observar en las imágenes obtenidas (Fig. 6), es una estructura ubicada en la base del cerebro en la que se unen las arterias carótidas internas antes de irrigar el parénquima cerebral. Debido a este tipo de morfología, la anastomosis o puente intercarotídeo permite que la circulación sanguínea se difunda de manera bilateral hacia ambos hemisferios. Consideramos que este tipo único de microcirculación arterial en las aves, les proporciona ciertas características de resistencia y protección ante eventos vasculares cerebrales, como isquemias experimentales o inducidas, debido a la compensación contralateral inmediata que tendría ante la falta de irrigación. Por lo tanto, coincidimos con lo demostrado por Frank \& Faraci (1991), quienes demostraron que las aves son más resistentes a eventos hipóxicos, debido a las características de la fisiología pulmonar, hemoglobina y transporte de oxígeno, vasculatura cerebral y efectos de constricción y vasodilatacíon así como a la morfología celular cerebral relacionada con la alta densidad de capilares.

Otra diferencia importante entre aves y mamíferos es la relacionada con la formación de la Arteria Basilar (AB), la cual se deriva de la Arteria o Rama Caudal Derecha (ACD), Aslan et al. 2006), describe que la arteria basilar puede estar formada por la Arteria o Rama caudal Izquierda en el faisán, y el pavo, mientras que en el gallo doméstico y el ganso deriva de la Rama Derecha, lo que coincide con nuestros hallazgos. Es importante mencionar la ausencia de las arterias vertebrales en el gallo doméstico.

Ante la elección de las aves como modelos experimentales en el área de las neurociencias, debe considerarse la importancia del reconocimiento de las estructuras vasculares cerebrales en las aves, poniendo especial énfasis en la anatomo fisiología de la Anastomosis Intercarotídea, Arteria Basilar y ausencia de Arterias Vertebrales. 
Con respecto a una posible contribución o proposición de este trabajo a la nomenclatura de las estructuras anatómicas y vasculares, consideramos que es necesario unificar la nomenclatura de las diversas estructuras anatómicas y vasculares. Ebert 2005, plantea que las estructuras cerebrales de las aves, deben ajustarse a una nueva nomenclatura, debido a que el vocabulario tradicional debe actualizarse ya que no concuerda con los actuales hallazgos neurofisiológicos en las aves. Llama la atención que en las descripciones anatómicas realizadas en las revistas científicas y en los atlas de anatomía de las aves, existe cierta confusión, ya que la nomenclatura de las estructuras no es clara y varía incluso entre un esquema y otro Ghetie, V.1981, Sisson, J. \& J. Grossman.1983. Por ejemplo, en el artículo de Aslan et al. (2006), se menciona que una de las anastomosis extracraneales es la formada por las arterias oftalmotemporal y la Arteria Carótida Cerebral, sin embargo, cabe aclarar que la arteria oftalmo temporal es extracraneal, y la Arteria Carótida Cerebral, no aclara si es carótida interna o externa, por lo tanto solo se podría interpretar que irriga el parénquima cerebral, es así, que la nomenclatura no es confiable.

Podríamos considerar como aportación al trabajo lo siguiente: Tomando en cuenta que las Arterias Carótidas Internas derivan de las Arterias Carótidas Comunes, y las Carótidas internas forman en la base del cerebro a la AI, las arterias que se forman o derivan después de la AI podrían considerarse como Arterias Cerebrales ya que estas prefunden directamente sobre el parénquima cerebral, lo cual puede apreciarse en la Figura 5 y 6.La referencia de la AI es un punto ideal de referencia para homogeneizar la nomenclatura.

(Pettit et al.1981), aporta otro hallazgo interesante con respecto a la función a anatomía de la RMO cuya función es mantener las diferencias de temperatura entre el cerebro y el cuerpo, lo cual ha sido documentado en diferentes reportes (Kilgore et al. 1979, Bernstein et al. 1979a, 1979b).

(Pettit et al ) señala que en las aves marinas la ACComún suministra el flujo sanguíneo por medio de la AOExterna a la RMO, hallazgo que no ocurre en las aves domésticas donde la AOExterna deriva de la ACInterna. La creación de una nueva nomenclatura permitirá replantear estudios y equiparar u homologar aspectos anatómicos, fisiológicos, así como el estudio de habilidades de tipo cognitivo entre el cerebro de aves y mamíferos.

Finalmente, proponemos el uso de técnicas imagenológicas como la resonancia magnética, angiografías, tomografía axial, así como la aplicación de estudios neuroquímicos, mediante los cuales enriquecerán las áreas de investigación referentes a la vasculatura cerebral en aves, lo anterior, basándose en que no hay material suficiente que describa la neuroanatomía vascular de las aves, exceptuando el trabajo realizado por Holliday et al. (2006) en el cual se utilizó equipo de resonancia magnética. El estudio integral del cerebro de las aves, y los nuevos 
hallazgos, permitirá que los diversos trabajos de investigación puedan homologarse entre aves y mamíferos.

La teoría de que el cerebro de las aves es el más primitivo debe ser replanteada, ya que el funcionamiento neurofisiológico de estas especies puede ser aún más complejo. En base a los actuales hallazgos anatómicos neurofisiológicos y bioquímicos del cerebro de las aves esta teoría debe quedar a consideración.

\section{LITERATURA CITADA}

Abdalla, M.A. \& A.S. King. 1977. The avian bronchial arteries: species variations. Journal of Anatomy, 123(3): 697-704.

Abdel-Magied E.M. \& A.S. King. 1978. The topographical anatomy and blood supply of the carotid body region of the domestic fowl. Journal of Anatomy, 126 (3): 535-546.

Aslan, K., H. Atalgin., I. Kürtül \& E.U. Bozkurt. 2006. Patterns of the internal and cerebral carotid arteries in various avian species: a comparative study. Revue de Médecine Vetérinaire, 157(12): 621624.

Baumel, J.J., \& L. Gerchman. 1968. The avian intercarotid anastomosis and its homologue in other. vertebrates. The American Journal of Anatomy, 122: 1-18.

Bernstein, M.H., M.B. Curtis \& D.M. Hudson. 1979b. Independence of brain and body temperatures in flying American Kestrels, Falco sparerius. American Journal of Physiology, 237: R58-F62.

Bernstein, M.H., I.V. Sandoval., M.B. Curtis \& D.M. Hudson. 1979a. Brain temperature in pigeons: effects of anteriorespiratory bypass. Journal Comparative Physiology, 129: 115-118.

Claus, J. 2001. Selective brain cooling in mammals and birds. Japanese Journal of Physiology, 51: 291301.

Ebert, J. 2005. Reformation of bird-brain terminology takes off. Nature, 433(7025): 449.

Faraci, F.M. 1991. Adaptations to hypoxia in birds: how to fly high. Annual Review of Physiology, 53: 59-70.

Ghetie, V. S. T. Chitescu., V. Cotofan \& A. Hillebrand. 1981. Atlas de Anatomía de las Aves Domésticas. Ed. ACRIBIA. Zaragoza, España. pp. 195-208.

Glenny, F. H. 1945a. A Systematic Study of the Main Arteries in the Region of the Heart-Aves XV: Gaviiformes-Part 1. The Ohio Journal of Science, 45(4): 167-169.

Glenny, F. H. 1945b. A Systematic Study of the Main Arteries in the Region of the Heart-Aves XXI: Passeriformes-Paridae. Part 1 The Ohio Journal of Science, 45(1): 19-21.

Glenny, F. H. 1951. A Systematic Study of the Main Arteries in the Region of the Heart-Aves XVII: Psittaciformes, Part I. The Ohio Journal of Science, 51(6): 347-352.

Glenny, F. H. 1951. A Systematic Study of the Main Arteries in the Region of the Heart-Aves XII: Galliformes, Part 1.The Ohio Journal of Science, 51(1): 47-54.

Glenny, F. H. 1953. A Systematic Study of the Main Arteries in the Region of the Heart Aves XIX: Apodiformes, Part 1. The Ohio Journal of Science, 53(6): 367-369.

Holliday, C. M., R. C. Ridgely., A.M. Balanoff \& L.M. Witmer. 2006. Cephalic Vascular Anatomy in Flamingos (Phoenicopterus ruber) Based on Novel Vascular Injection and Computed Tomographic Imaging Analyses. The Anatomical Record Part A. 288A: 1031-1041.

Kilgore, D. L., D. F. Boggs, \& G. F. Birchard. 1979. The role of the Rete Mirable Ophthalmicum in maintaining the body-to-brain temperature difference in pigeons. Journal Comparative Physiology, 129: 119-122.

McLelland J. 1992. Atlas en color de Anatomía de las aves. Interamericana-Mc Graw-Hill. España. 
Morales-Martínez, J.J., G.F. Ayala \& L.A. Poblano. 2002. Diferencias auditivas del tallo cerebral en el gallo y el cuy, Estudio electrofisiológico comparativo de los potenciales provocados auditivos tempranos. Acta Zoológica Mexicana (n.s.), 87: 83-97.

Pettit, T.N., G. Causey-Whittow, \& Grant, S.G. 1981. Rete Mirabile Ophthalmicum in Hawaiian Seabirds. The Auk, 98: 844-846.

Sisson, J. \& J. Grossman.1983. Anatomía comparada de los animales domésticos. Salvat. México. 